\title{
A robust approach for WSN localization for underground coal mine monitoring using improved RSSI technique
}

\author{
Banda Srikanth $^{1 *}$, Hemant Kumar' ${ }^{2}$ Karanam U.M Rao ${ }^{3}$ \\ ${ }^{1}$ University College of Engineering, Kakatiya University, Kothagudem, Telangana State 507101, India \\ ${ }^{2}$ Rock Mechanics and Underground Metal Mining, Department of Mining Engineering, IIT(ISM) Dhanbad 826004, India \\ ${ }^{3}$ Department of Mining Engineering, Indian Institute of Technology, Kharagpur 721302, India
}

Corresponding Author Email: bsrikanthiitkgp@gmail.com

https://doi.org/10.18280/mmep.050314

Received: 11 July 2018

Accepted: 31 July 2018

\section{Keywords:}

WSN, coal mine monitoring, localization,

localization error, signal strength

\begin{abstract}
During last decade, wireless communication technologies have grown drastically and have been adopted widely for various real-time applications. Wireless sensor network based communication has attracted researchers' attention due to its significant nature of adaptability in diverse application. Wireless sensor networks are widely used in applications such as environment monitoring, military application, weather forecasting etc. However, numerous researches have been carried out in these monitoring systems. Recently, mining industries are also adopting WSN based communication for monitoring underground coal miners for worker safety purpose and better production. In order to achieve better convergence analysis of coal mine, a proper data transmission from sensor to the destination node is required, which is possible only if the accurate positions of sensor nodes are known. Finding the accurate position is a crucial task, which is also known as sensor node localization. In this study, we have focused on the underground coal mining monitoring, where first of all, we present a novel approach for sensor network localization to establish hassle free communication. According to proposed approach, initially a sensor network localization problem is formulated where node distance measurement and signal strength measurement models are developed. Later RSSI based signal strength model is developed, which is further improved by introducing a probability density function, which helps to reduce the distance error. Complete experimental study is carried out using MATLAB simulation tool where a scenario of underground coal mining is considered. Experimental study shows that proposed model outperforms Gaussian model and Statistical Mean Model by achieving average range error of 3.67, average localization error of 4.85 in terms of communication radius and average range error of 6.972 for varied number of nodes.
\end{abstract}

\section{INTRODUCTION}

In last two decades, the demand and applications of various communication systems have grown drastically. In this context of communication system, wireless communication is considered as a most promising technique of communication and it has significant impact on real-time communication systems [1]. Various researches have been presented to delineate the wireless communication which also embodies the discussion about advantages and disadvantages of wireless communication [2]. In this field of wireless communication, sensor network based communication plays important role and it is considered as an active area of research. These sensor nodes are used to formulate a sensor network, called as Wireless sensor network which utilizes small sensor nodes. Since, these sensor nodes are very small in size, hence, various limitations are also constrained such as limited memory capacity, battery capacity and processing time etc. [3]. Generally, these sensor networks are deployed in distributed manner for sensing the physical conditions of the outer world and monitor the environmental conditions such as light, weather conditions and pollution etc. These sensor networks enormously prolong the user's ability to monitor the environmental and physical activities. Moreover, wireless sensor networks are anticipated to accommodate the solution for various applications on real-time systems such as monitoring, military purpose, disaster management and patient tracking and monitoring etc. The augmented study of WSNs helps to serve communication better by introducing automated monitoring system [4]. Due to increased capacity of sensor nodes, these sensor nodes are used for wide range of applications such as agriculture, geological analysis, navigation purpose and security and along with this, these networks can be used for monitoring underground conditions [5]. Moreover, sensor networks are adopted for examine the conditions of underground infrastructures and process such as landslide detection, earthquake monitoring and information acquisition from the sensor network which is deployed in underground tunnels or mines [6].

In this work, we focus on the monitoring the underground environmental condition which can be used for various mining industries such as coal mining. Environment monitoring and efficient communication is considered as a crucial task which helps to ensure the safety in underground coal mines and improves the productivity. Generally, in underground coal mines, poor visibility, lower ventilation and toxic gases are 
present which may cause explosion resulting in huge damage and loss of capital and severe causalities. Industries urge for a significant system for provisioning advanced monitoring system which can accommodate real-time information about underground mines and miners, specifically it helps to procure the real-time location parameters of the miners which can be used for ensuring the safety of miners in emergency. In order to guarantee the safety in underground coal mining systems, Cable Monitoring System (CMS) has shown a significant effect on various issues [7] but due to complexity in underground environments, CMS technique fails to monitor the various parameters which have impact on the coal mining safety such as underground temperature, pressure, speed of wind and carbon monoxide level measurement. Moreover, it is difficult to identify the hidden dangers which become a complex task for CMS systems to overcome. In contrast, wireless sensor network based techniques can efficiently, insistently and flexibly monitor the underground mine areas which are not possible to measure by CMS systems. These systems have various limitations such as: these techniques are vulnerable to failure due to cable breakage, bad design or connectivity may lead to the sparks or flame resulting in fire, in wired systems connection cannot be established from the desired location wirelessly etc.

Generally, in dangerous situations such as disaster (fire, landslide), WSN can be considered as more reliable comparative to the wired communication systems. Wireless sensor network systems can be deployed rapidly and it has multi-hop transmission system which can provide more scalable and reliable information for underground monitoring. Furthermore, WSNs can be used to localize the miner in underground mines where other techniques require a predefined infrastructure for monitoring where GPS (Global Positioning Systems) are not available and implementation of GPS systems for underground coal mining becomes crucial due to implementation cost. However, WSN suffer from various issues which are responsible for affecting the performance of WSN such as medium access, deployment of sensor network, network layer, security and localization of sensor node. In this process of underground mine monitoring, sensor node localization considered as important task which helps to perform network routing, location awareness and location based tacking. In underground coal mining systems, obtaining the accurate information is a challenging task hence localization technique needs to be implemented for any underground WSN monitoring system which can provide the information about miners. According to WSN, any information is collected from source node and transmitted towards the destination nodes with the help of anchoring nodes. If the position of anchor node, source node and destination node is not known then the collected information may not be transmitted efficiently or information may become useless due to loss of the packets. In order to deal with this issue, GPS is a promising technique but implementation cost and complexity is a challenging task. Hence, we focus on the wireless sensor network based localization scheme. WSN localization techniques can be divided into two classes: anchor-based and anchor-free algorithm for WSN localization. According to anchor based approach, it is assumed that all reference nodes are anchor nodes whose positions and coordinates are known in advance whereas anchor-free localization algorithms need few anchor nodes where coordinates of these reference nodes are computed automatically.
These algorithms of WSN localization in underground coal mines may face various challenges due to water vapor and coal dust which may degrade the signal and lead to the localization error. Along with this, underground mines have complex structure due to that, a complex network topology require where conventional localization approach may fail to work. In order to deal with this issue, here we present a novel approach for WSN localization for underground coal mining. The main contributions of this work are as follows:

(a) First of all, we present distance measurement and signal strength measurement models

(b) In the next phase, RSSI based model is used for node localization measurement

(c) Finally, a probability density function based improved RSSI model is used to reduce the localization error.

Rest of the manuscript is organized as follows: a brief study about recent techniques in this field of underground coal mine monitoring is discussed in section II, section III presents proposed approach for sensor node localization, based on the proposed approach, we present comparative experimental analysis which is depicted in section IV and finally, section V presents concluding remarks regarding proposed approach.

\section{LITERATURE SURVEY}

In this section, we discuss about recent techniques of underground coal mining monitoring using wireless sensor network localization where multiple techniques of sensor network localization are discussed along with their key finding, advantages and disadvantages. Moridi et al. [8] et al. discussed about the underground monitoring and communication system for mining systems. Generally, underground mining systems are challenging to complex terrain and changing network topology may cause reliability and performance issues. In this work, automated process of monitoring is adapted using ZigBee networking process. This study shows the advantages of ZigBee for monitoring of underground mine. Moreover, ZigBee radio waves attenuation is also analyzed which helps to find the suitable distance for ZigBee node placement for curved and straight tunnels. It shows better performance but the main limitation of this work is that it can be implemented for short range of underground monitoring and the cost of implementation also becomes a challenging task. Wang et al. [9] presented a novel combined approach for underground monitoring using the ZigBee and Wi-Fi communication models. The main aim of this work is to divide the study based on the rational utilization of network resources where different data types can be transmitted using ZigBee of Wi-Fi techniques. According to this process, the data which doesn't contain any video, audio and image type of information; will be transmitted through the ZigBee network and the data with video and audio will be transmitted through Wi-Fi network. This realization of data transmission helps to preserve the energy for transmission and helps to reduce the energy consumption during data transmission. Similarly, Bedford et al. [10] evaluated the issues of underground mine monitoring and presented low cost ZigBee based modules for position estimation using radio frequency (RF) techniques. This work is considered for two tunnel scenarios where various conditions are present for location based technique. In order to estimate the accurate position, time of flight measurement technique is used. This study shows that RF waves experiences 
various interferences from the microwave type of networks which are deployed in mines.

These localization and underground coal mine monitoring systems which are based on the ZigBee communication, RF communication and $\mathrm{Wi}-\mathrm{Fi}$ can be adopted for coal mine monitoring purpose but the implementation cost and complexity remains an unaddressed issue in this field. Recently, Qin et al. [11] presented Ultra Wideband (UWB) communication technology for underground coal mine monitoring. In underground mine monitoring scenario diffraction, reflection and diffusion on sidewall causes measurement error. Hence, authors discussed about UWB systems which are capable to provide precise ranging for accurate measurement using time of flight measurement technique. Prior to this work of [11], Chehri et al. [12] also discussed about the significance of UWB for real-time communication systems. UWB systems have several advantages such as low cost of implementation, low complexity and significant nature to provide time domain resolution which helps to achieve location estimation and tracking applications. This work aims on the characterization of underground mines, ToA estimation; location based fingerprinting and positioning algorithms.

In this field of underground coal mine monitoring, wireless sensor networks also play important role for monitoring the conditions of mines and providing safety to the miners. Based on the concept of wireless sensor network communication, $\mathrm{Li}$ et al. [13] presented WSN based coal mine monitoring system where a Structure-Aware Self-Adaptive sensor network is developed. In this process the sensor network is deployed in mesh network form and beacon strategy is used for establishing the communication. Moreover, this system is robust for managing the queries in various circumstances where environmental conditions are changing. This study shows that use of wireless sensor network can provide better performance for underground coal mine monitoring system when compared with other wireless communication technologies. Several researches have been presented for underground coal mining using wireless sensor network technique. Wu et al. [14] presented a comprehensive approach for routing in underground coal mine environment using WSN Conventional protocols of WSN are built on the layered protocol architecture for dividing the complexity based on each layer but optimal performance becomes a challenging task in layered protocol. To overcome this issue, authors presented a holistic approach for underground communications. For underground coal mining monitoring and safety purpose, Bhattacharjee et al [15] presented a novel approach which is used for fire detection, monitoring and alarming purpose. This study is carried out for Bord-and-Pillar coal mine panel using WSN which helps to identify the location of fire and its spreading direction. Moreover, it helps to prevent the fire spreading with the help of early detection of fire.

Chen et al. [16] discussed about coal mining monitoring system and analyzed that coal mine tunnels have a good spatial-temporal correlation. In this work, authors focused on the energy consumption reduction and efficiency improvement for network monitoring of underground tunnels. Hence, network node correlation based method is used along with Bayesian decision formulation. Based on the movement of the miners, a choice is made between tunnels and pathways and Bayesian decision based technique is used for prediction of path in the underground environment. Recently, Zhou et al.
[17] discussed about coal mine tunnel monitoring using wireless sensor networks. Proper deployment of sensor node is mostly demanded in WSN which can help to obtain the significant information. To deal with this, 3D band-type node deployment process is implemented where various features and characteristics are considered such as sensing efficiency, coverage feature, redundancy principles and radio features. In next phase, target node deployment is discussed and finally, simulated annealing based optimization scheme is discussed for providing optimal performance.

This section presents a brief study about the recent techniques of underground coal mine monitoring. In this study, we discussed about several techniques where some of the techniques are based on the ZigBee communication, Wi-Fi communication and RF etc. These have been widely adopted for monitoring the underground mines but due to complex terrain and underground tunnel complexities, these techniques fail to provide the accurate monitoring. To overcome this issue, sensor networks technique is introduced which shows a significant improvement in underground monitoring systems. However, if the location of source, anchor and destination node is not known then the collected information may lose resulting in inaccurate monitoring hence, sensor network localization is an important task for achieving better performance of underground coal mine monitoring systems.

\section{PROPOSED MODEL}

In this section, we present the proposed model for sensor node localization for underground coal mining applications. The complete section is divided into three main parts: (a) problem formulation where distance measurement and signal strength measurement based problem formulation is modeled based on maximum likelihood method. (b) In next phase, we apply maximum likelihood which helps to reduce the mean square error. In this phase, maximum likelihood matrix selection method and finally (c) we present maximum likelihood model for finding the maximum probable value from the previously obtained result.

Let us consider that a set of known position are given in $m-$ dimensional vector given as $x_{1}, x_{2} \ldots, x_{N}$ and unknown single source location is given by $y$ and based on the proposed approach, the estimated source location is denoted by $\hat{y}$.

\subsection{Node distance measurement model}

In this model, we measure the distance from source node to each destination node which can be computed as:

$d_{i}^{2}=\left\|k_{i}-y\right\|^{2}+\mathbb{N}_{i}$

where $\mathbb{N}_{i}$ denotes the noise which is distributed identically with zero mean and $\sigma^{2}$ variance. For this measurement, the conditional probability density function can be expressed as:

$\mathcal{P}\left(d_{1}^{2}, d_{2}^{2}, \ldots, d_{N}^{2} \mid y\right)=\frac{1}{\left(2 \pi \sigma^{2}\right)^{\frac{N}{2}}} e^{-\frac{\sum_{i=1}^{N}\left(d_{i}^{2}-\left\|k_{i}-y\right\|^{2}\right)^{2}}{2 \sigma^{2}}}$

And the maximum likelihood of this distance measurement can be computed as: 
$\hat{y}=\underset{y}{\arg \min } \sum_{i=1}^{N}\left(d_{i}^{2}-\left\|k_{i}-y\right\|^{2}\right)^{2}$

\subsection{Measurement of signal strength}

Here we present a model for measuring the signal strength during data transmission. Let us consider that source node $y$ transmits a signal with $P$ power then sensor at location $k_{i}$ can receive a signal power $\mathcal{S}_{i}$ as:

$\delta_{i}=P\left\|k_{i}-y\right\|^{-\beta}$

where $\beta$ denotes the path loss coefficient, by considering a log-normal fading, the received signal strength at any sensor node can be computed as:

$10 \log S_{i}=10 \log P-10 \log \left(\left\|k_{i}-y\right\|\right) \beta+\mathbb{N}_{i}$

where $\mathbb{N}_{i}$ denotes the noise which is distributed identically with zero mean and $\sigma^{2}$ variance. Similar to the node distance computation model, here we compute probability density function, which can be computed as:

$$
\begin{aligned}
& \mathcal{P}\left(\ln \mathcal{S}_{1}, \ln \mathcal{S}_{2}, \ldots, \ln \mathcal{S}_{N} \mid y\right)= \\
& \frac{1}{\left(2 \pi\left(\ln \frac{10}{10 . \sigma}\right)^{2}\right)^{N / 2}} e^{-\frac{\sum_{i=1}^{N}\left(\ln \mathcal{S}_{i}-\ln \left(\frac{P}{\left\|k_{i}-y\right\|^{\beta}}\right)\right)^{2}}{2\left(\ln \frac{10}{10 . \sigma}\right)^{2}}}
\end{aligned}
$$

Based on this model, the maximum likelihood function can be expressed as:

$\hat{y}=\underset{y}{\arg \min } \sum_{i=1}^{N}\left(\ln \mathcal{S}_{i}-\ln \left(\frac{P}{\left\|k_{i}-y\right\|^{\beta}}\right)\right)^{2}$

\subsection{RSSI based ranging model}

Previous sub-section described the problem formulation for distance measurement and signal strength measurement with the help of probability density function and maximum likelihood computation. In this section, we present the ranging model for underground coal mining system using RSSI (Received Signal Strength Indicator). As discussed before, that sensor nodes localization can be divided into two categories where coordinates of reference nodes are known initially and the location of blind node need to identify. Reference nodes are also known as anchor nodes which are placed in the known location and these nodes are responsible for broadcasting the signal to surrounding space in a fixed range of frequency. However, these signals carry various information along with the information about their own coordinate. Later, unknown node receives this information i.e. RSSI from the anchor nodes and performs the coordinate computation by using localization algorithms.

In this process of data transmission, transmission node and receiving node require some power. The relationship between transmitting node power $\left(T_{x}\right)$ and receiving node power $\left(R_{x}\right)$ of transmitting signal can be given as:

$P_{T x}=d^{n} \cdot P_{R x}$

where $P_{R x}$ denotes the power of receiver node, $P_{T x}$ is used for transmitter node power and the distance between these nodes is given by $d$ and $n$ denotes the path loss factor which depends on the condition of underground environment. The power required for data transmission and receiving, it should be measured in $\mathrm{dB}$ hence we apply logarithmic computation on both side Eq. (8) and it can re-write as:

$10 \log P_{R x}=10 \log P_{T x}-10 n \log d$

$10 \log P_{R x}$ denotes the required power expression in $\mathrm{dB}$ and it can be called as RSSI and let us denote the $T x$ power with a constant value and the RSSI based ranging mode can be expressed as:

$\operatorname{RSSI}(d B)=C-10 n \log d$

Generally, the value of C is considered the value of RSSI computed at 1 meter away from the transmitting node. The value of final RSSI depends on the distance if the distance is increasing then the RSSI value will decrease.

Let us consider that the anchor nodes are given as $A_{1}, A_{2}, \ldots, A_{n}$ and according to the coordinate system, the location of these nodes can be expressed as $\left(x_{A 1}, y_{A 1}\right),\left(x_{A 2}, y_{A 2}\right), \ldots,\left(x_{A n}, y_{A n}\right)$. It is assumed that blind or unknown node $(U)$ coordinates are $(x, y)$. Based on this, distance between unknown node and reference node can be computed as:

$\left\{\begin{array}{l}d_{1}^{2}=\left(x_{A 1}-x\right)^{2}+\left(y_{A 1}-y\right)^{2} \\ d_{2}^{2}=\left(x_{A 2}-x\right)^{2}+\left(y_{A 2}-y\right)^{2} \\ d_{n}^{2}=\left(x_{A n}-x\right)^{2}+\left(y_{A n}-y\right)^{2}\end{array}\right.$

From node 1 to $(n-1)$ node it subtracts and it can be represented such as:

$$
\begin{gathered}
2 x\left(x_{A n}-x_{A 1}\right)+2 y\left(y_{A n}-y_{A 1}\right)=d_{1}^{2}-d_{n}^{2}-\left(x_{A 1}^{2}+\right. \\
\left.y_{A 1}^{2}\right)+\left(x_{A n}^{2}+y_{A n}^{2}\right) \\
2 x\left(x_{A n}-x_{A 2}\right)+2 y\left(y_{A n}-y_{A 2}\right) \\
=d_{2}^{2}-d_{n}^{2}-\left(x_{A 2}^{2}+y_{A 2}^{2}\right)+\left(x_{A n}^{2}+y_{A n}^{2}\right) \\
\vdots \\
2 x\left(x_{A n}-x_{A(n-1)}\right)+2 y\left(y_{A n}-y_{A(n-1)}\right)=d_{n-1}^{2}-d_{n}^{2} \\
-\left(x_{A(n-1)}^{2}+y_{A(n-1)}^{2}\right)+\left(x_{A n}^{2}+y_{A n}^{2}\right) .
\end{gathered}
$$

In other terms, this equation can be expressed as $C X=b$ where $X=\left(\begin{array}{l}x \\ y\end{array}\right)$,

$$
\begin{gathered}
C=\left(\begin{array}{cc}
2\left(x_{A 1}-x_{R n}\right)+ & 2\left(y_{A 1}-y_{A n}\right) \\
\cdots & \ldots \\
2\left(x_{A(n-1)}-x_{R n}\right)+ & 2\left(y_{A(n-1)}-y_{R n}\right)
\end{array}\right) \text { and } \\
b=\left(\begin{array}{c}
x_{A 1}^{2}-x_{A n}^{2}+y_{A 1}^{2}-y_{A n}^{2}+d_{n}^{2}-d_{1}^{2} \\
\cdots \\
x_{A(n-1)}^{2}-x_{A n}^{2}+y_{A(n-1)}^{2}-y_{A n}^{2}+d_{n}^{2}-d_{(n-1)}^{2}
\end{array}\right)
\end{gathered}
$$

Based on this RSSI ranging model, distance values $\left(d_{1}, d_{2}, \ldots, d_{n}\right)$ can be computed Equation, $C X=b$ can be solved with the help of MMSE computation and the coordinates of unknown node can be given as:

$X=\left(C^{T} C\right)^{-1} C^{T} b$ 


\subsection{Improved localization approach using probability density function}

In wireless sensor network based communication, the senor data is transmitted to the server or destination node. In this work, we use maximum likelihood techniques for location estimation with the help of anchor nodes. This technique provides the maximum probable value for better location estimation by utilizing previously obtained values. A probability density function is formulated as discussed in previous sections. Based on these probability values we determine whether the current coordinates of node belong to which anchor node. In order to perform this, first of all we formulate trilateration group which is denoted by $\mathcal{L}_{k}, k=$ $1,2,3 \ldots L$ where $L$ denotes the total number of trilateration group available. To determine the closest relationship of coordinates $(\alpha)$ with the anchor node, we compute the conditional probabilities as:

$p\left(\mathcal{L}_{k} \mid \alpha\right), k=1,2,3, \ldots L$

This conditional probability value $p\left(\mathcal{L}_{k} \mid \alpha\right)$ provides the relationship of $\alpha$ coordinates with the reference node and determines whether estimated locations belong to the correct trilateration group. These anchor nodes can be categorized using decision rule as:

$\alpha \in \mathcal{L}_{k}$ if $p\left(\mathcal{L}_{k} \mid \alpha\right)>p\left(\mathcal{L}_{n} \mid \alpha\right)$ for all $n \neq k$

This decision rule helps to correlate between desired $p\left(\mathcal{L}_{k} \mid \alpha\right)$ and projected data $p\left(\mathcal{L}_{n} \mid \alpha\right)$ using Bayes computation as:

$p\left(\mathcal{L}_{k} \mid \alpha\right)=\frac{p\left(\alpha \mid \mathcal{L}_{k}\right) p\left(\mathcal{L}_{k}\right)}{p(\alpha)}$

$\mathcal{L}_{k}$ is the probability of movement of anchor node from its trilateration group, $p(\alpha)$ is the final probability to identify the anchor node with the reference location which can be given as: $p(\alpha)=\sum_{k=1}^{L} p\left(\alpha \mid \mathcal{L}_{k}\right) p\left(\mathcal{L}_{l}\right)$. With the help of (16), Eq. (15) can be re-written as:

$$
\alpha \in \mathcal{L}_{k} \text { if } p\left(\alpha \mid \mathcal{L}_{k}\right) p\left(\mathcal{L}_{k}\right)>p\left(\alpha \mid \mathcal{L}_{n}\right) p\left(\mathcal{L}_{n}\right) \text { for all } n \neq k
$$

Based on this decision rule, final probabilities are computed and sorted based in descending order for current reference/ anchor node.

\section{EXPERIMENTAL RESULTS}

This section provides the detailed description of experimental study for underground coal mining sensor network localization. We used MATLAB simulation tool to carry out the experimental study. in this scenario, we have considered a tunnel type of track with size of $100 \mathrm{mX} 100 \mathrm{~m}$ where multiple sensor nodes are deployed. Initial coordinates are considered as $(0,0)$ and the path attenuation factor is $n$ considered as 3.5 , A Gaussian distribution with 0 mean is considered here along with a standard deviation of 6 . It is considered that miners are moving with a constant speed of four beacon nodes. Based on these assumptions, we compute the RSSI based performance measurement and compared the localization performance with the proposed approach. A network deployment model is given in figure 1 .

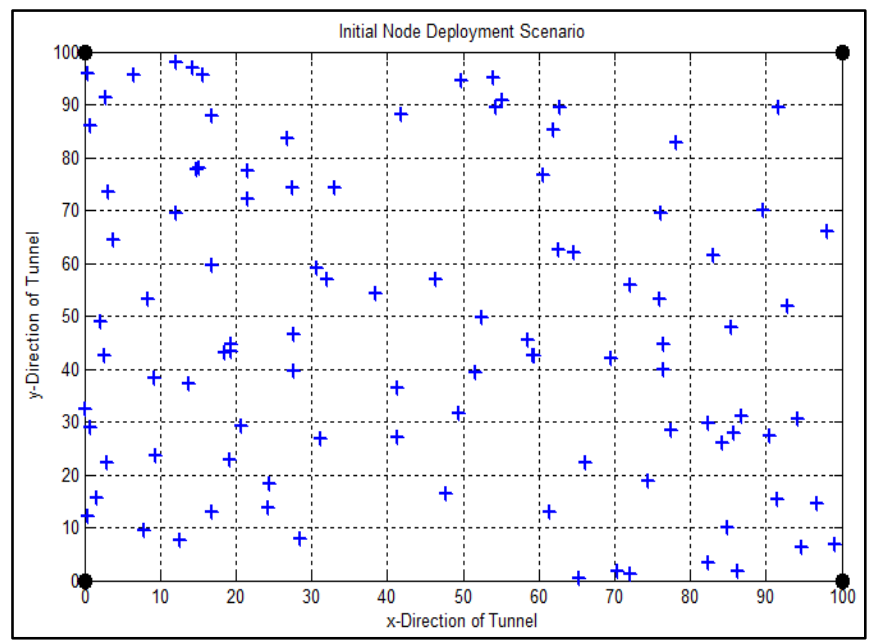

Figure 1. Initial node deployment

In this case, we have considered varied numbers of communication range to estimate the RSSI based ranging model. In order to measure the performance of proposed approach, ranging error is computed. The communication range is varied from 5 meters to 45 meters. Figure 2 shows sensor node localization performance for 100 nodes scenario where 4 anchor nodes are present.

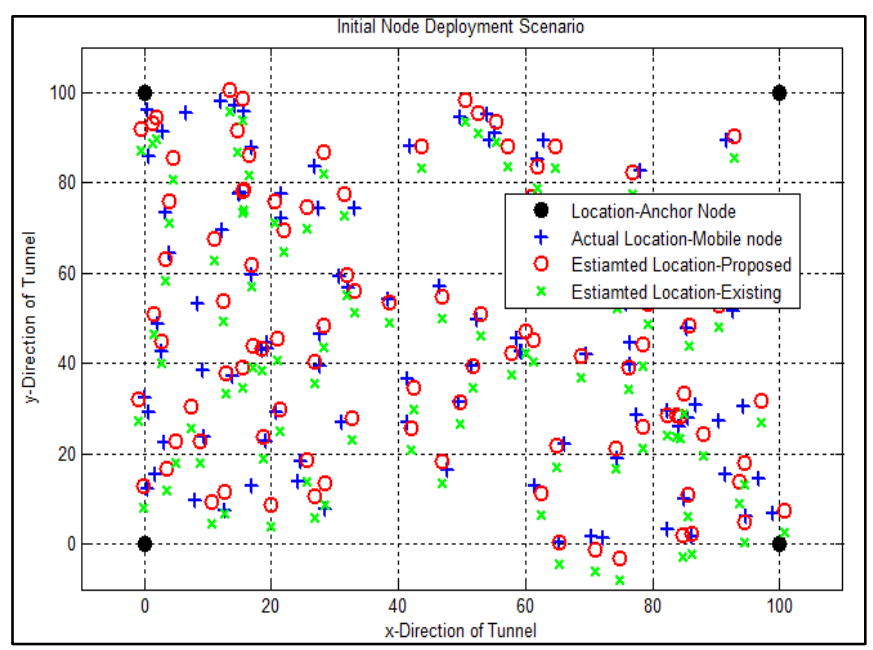

Figure 2. Sensor node localization

Based on this localization study, we compute ranging error performance and compared the performance of RSSI based Gaussian model, Statistical Mean model and proposed approach. This performance is depicted in figure 3 .

According to the ranging error performance, average error using Gaussian model is obtained as 5.56, average error of statistical mean model is 4.71 and proposed model achieves average error of 3.67 which shows better improvement in range error reduction. The detailed performance is given in table 1. This experiment shows that the proposed approach achieves better performance when compared with the state-ofart techniques. Proposed approach achieves 33\% percent better performance when compared with the Gaussian model and $22 \%$ improvement when compared with the statistical mean model of localization. 


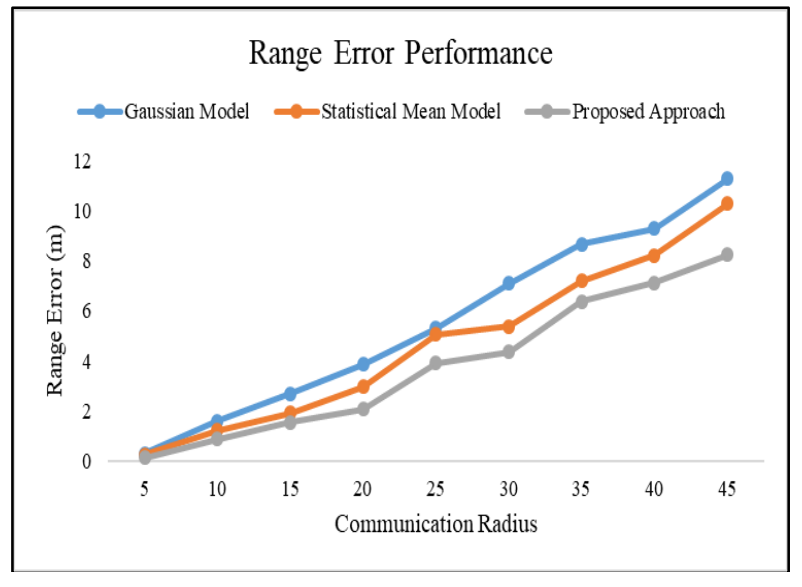

Figure 3. Range error performance

Table 1. Range error performance

\begin{tabular}{|c|c|c|c|}
\hline $\begin{array}{c}\text { Communicati } \\
\text { on Radius } \\
\text { Range }\end{array}$ & \multicolumn{3}{|c|}{ Range Error } \\
\hline & $\begin{array}{c}\text { Gaussian } \\
\text { Model }\end{array}$ & $\begin{array}{c}\text { Statistical Mean } \\
\text { Model }\end{array}$ & $\begin{array}{c}\text { Proposed } \\
\text { Approach }\end{array}$ \\
\hline 5 & 0.3 & 0.25 & 0.12 \\
\hline 10 & 1.6 & 1.22 & 0.87 \\
\hline 15 & 2.68 & 1.92 & 1.53 \\
\hline 20 & 3.86 & 2.96 & 2.06 \\
\hline 25 & 5.29 & 5.04 & 3.26 \\
\hline 30 & 7.09 & 5.37 & 4.11 \\
\hline 35 & 8.66 & 7.2 & 5.9 \\
\hline 40 & 9.28 & 8.21 & 7.11 \\
\hline 45 & 11.27 & 10.27 & 8.01 \\
\hline Average Error & 5.56 & 4.71 & 3.67 \\
\hline
\end{tabular}

In next case, we measure the range error performance by varying the number of nodes where total number of nodes are varied up to 500 nodes. Performance for varied number of nodes is depicted in figure 4 .

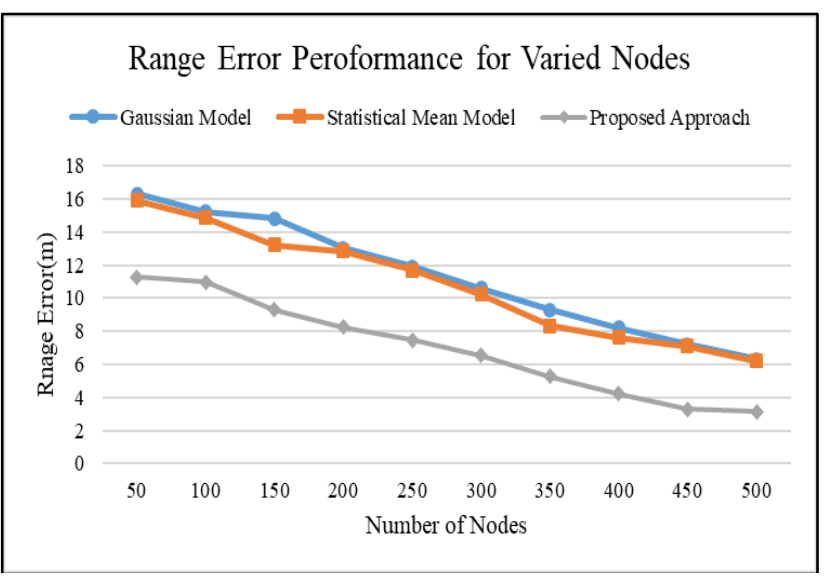

Figure 4. Range error performance for varied nodes

Similarly, we computed the performance of proposed approach for varied number of nodes and presented a comparative study in terms of range error. Average error using proposed technique is obtained as 6.97, Gaussian model as 11.30 and statistical mean model is 10.79 . Thus, this study shows that proposed approach achieves better performance in reducing the ranging error. According to this experiment, proposed approach shows $38.31 \%$ and $36.46 \%$ improvement when compared with the Gaussian and statistical mean model respectively.

Table 2. Range error performance for varied number of nodes

\begin{tabular}{|c|c|c|c|}
\hline $\begin{array}{c}\text { Number } \\
\text { of Nodes }\end{array}$ & \multicolumn{3}{|c|}{ Range Error } \\
\hline & $\begin{array}{c}\text { Gaussian } \\
\text { Model }\end{array}$ & $\begin{array}{c}\text { Statistical Mean } \\
\text { Model }\end{array}$ & $\begin{array}{c}\text { Proposed } \\
\text { Approach }\end{array}$ \\
\hline 50 & 16.3 & 15.89 & 11.27 \\
\hline 100 & 15.22 & 14.86 & 10.99 \\
\hline 150 & 14.84 & 13.2 & 9.28 \\
\hline 200 & 13.02 & 12.83 & 8.26 \\
\hline 250 & 11.92 & 11.67 & 7.46 \\
\hline 300 & 10.6 & 10.22 & 6.54 \\
\hline 350 & 9.3 & 8.33 & 5.28 \\
\hline 400 & 8.223 & 7.6 & 4.23 \\
\hline 450 & 7.21 & 7.11 & 3.29 \\
\hline 500 & 6.33 & 6.21 & 3.12 \\
\hline Average & 11.30 & 10.79 & 6.97 \\
\hline
\end{tabular}

Finally, we present the performance of proposed system in terms of localization error based on the communication radius as discussed before. A comparative performance is presented in figure 5 where proposed approach is compared with the existing techniques.

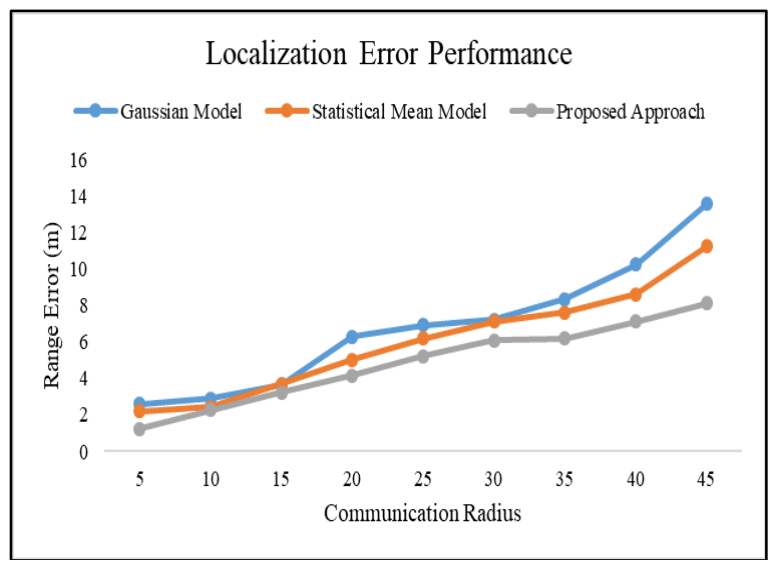

Figure 5. Localization error performance for varied range of communication

Table 3 demonstrates the localization error performance comparison. Here, the average error using proposed technique is obtained as 4.85, Gaussian model as 6.85 and statistical mean model as 6.00. Thus, this study shows that proposed approach achieves better performance in reducing the ranging error. Experimental study shows that proposed approach achieves $29.19 \%$ and $19.16 \%$ better performance when compared with the Gaussian model and statistical mean model. This performance is measured in terms of localization error reduction.

The above mentioned experimental study shows a comparative performance analysis for sensor node localization in underground coal mining systems. This performance measurement is carried out in various terms such as localization error and range error etc. 
Table 3. Localization error performance comparison

\begin{tabular}{|c|c|c|c|}
\hline $\begin{array}{c}\text { Communication } \\
\text { Radius Range }\end{array}$ & \multicolumn{3}{|c|}{$\begin{array}{c}\text { Localization Error Performance } \\
\text { comparison }\end{array}$} \\
\hline & $\begin{array}{c}\text { Gaussian } \\
\text { Model }\end{array}$ & $\begin{array}{c}\text { Statistical } \\
\text { Mean Model }\end{array}$ & $\begin{array}{c}\text { Proposed } \\
\text { Approach }\end{array}$ \\
\hline 5 & 2.6 & 2.2 & 1.23 \\
\hline 10 & 2.9 & 2.45 & 2.28 \\
\hline 15 & 3.66 & 3.69 & 3.23 \\
\hline 20 & 6.3 & 5.01 & 4.16 \\
\hline 25 & 6.92 & 6.2 & 5.22 \\
\hline 30 & 7.21 & 7.1 & 6.1 \\
\hline 35 & 8.33 & 7.6 & 6.2 \\
\hline 40 & 10.23 & 8.6 & 7.13 \\
\hline 45 & 13.56 & 11.23 & 8.12 \\
\hline Average Error & 6.85 & 6.00 & 4.85 \\
\hline
\end{tabular}

\section{CONCLUSIONS}

In this work, we have focused on wireless communication and used wireless sensor network based communication. WSN communication is introduced for underground coal mine monitoring purpose. In order to monitor the coal mine roof convergence, first of all we develop a methodology for better communication for underground coal mine to earth base station. In order to perform this task, we present WSN localization scheme using RSSI based distance measurement model which is further improved by introducing a probability density function which provides robust values of node coordinates for finding the accurate position of communicating nodes. An extensive experimental study is carried out by considering an underground coal mining scenario where multiple sensors are deployed and localization scheme is implemented. Experimental study achieves better performance in terms of localization error.

\section{REFERENCES}

[1] Yetgin H, Cheung KTK, El-Hajjar M, Hanzo LH. (2017). A survey of network lifetime maximization techniques in wireless sensor networks. IEEE Communications Surveys \& Tutorials 19(2): 828-854. https://doi.org/10.1109/COMST.2017.2650979

[2] Tse D, Viswanath P. (2005). Fundamentals of wireless communication. Cambridge university press.

[3] Borges LM, Velez FJ, Lebres AS. (2014). Survey on the characterization and classification of wireless sensor network applications. IEEE Communications Surveys \& Tutorials 16(4): 1860-1890. https://doi.org/10.1109/COMST.2014.2320073

[4] Mao C. (2017). Design and implementation of a WSNbased monitoring system for hazardous gas in chemical production. Chemical Engineering Transactions 59: 577582. https://doi.org/10.3303/CET1759097
[5] Shen S, Yang B, She Y, Jiang X. (2017). Research on the key technology of the internet of things in mine based on wireless Sensor. Chemical Engineering Transactions 62: 829-834. https://doi.org/10.3303/CET1762139

[6] Akyildiz IF, Stuntebeck EP. (2006). Wireless underground sensor networks: Research challenges. Ad Hoc Networks 4(6): 669-686. https://doi.org/10.1016/j.adhoc.2006.04.003

[7] Ni LM, Liu Y, Zhu Y. (2007). China's national research project on wireless sensor networks. IEEE Wireless Communications 14(6): 78-83.

[8] Moridi MA, Kawamura Y, Sharifzadeh M, Chanda EK, Jang H. (2014). An investigation of underground monitoring and communication system based on radio waves attenuation using ZigBee. Tunnelling and Underground Space Technology 43: 362-369. https://doi.org/10.1016/j.tust.2014.05.011

[9] Wang LN, Huang YR, Tang CL, Qu LG. (2014). Design and realization of underground monitoring and control system based on Zigbee+ Wifi. Coal engineering 46(6): 138-140.

[10] Bedford MD, Kennedy GA. (2012). Evaluation of ZigBee (IEEE 802.15. 4) time-of-flight-based distance measurement for application in emergency underground navigation. IEEE Transactions on Antennas and Propagation 60(5): 2502-2510. https://doi.org/10.1109/TAP.2012.2189731

[11] Qin Y, Wang F, Zhou C. (2015). A distributed UWBbased localization system in underground mines. Journal $\begin{array}{lll}\text { of } & \text { Networks } & 134(3) \text { : }\end{array}$ https://doi.org/10.4304/jnw.10.3.134-140

[12] Chehri A, Fortier P, Tardif PM. (2009). UWB-based sensor networks for localization in mining environments. Ad Hoc Networks 7(5): 987-1000. https://doi.org/10.1016/j.adhoc.2008.08.007

[13] Li M, Liu Y. (2009). Underground coal mine monitoring with wireless sensor networks. ACM Transactions on $\begin{array}{llll}\text { Sensor } & \text { Networks } & \text { (TOSN) }\end{array}$ https://doi.org/10.1145/1498915.1498916

[14] Wu D, Bao L, Li R. (2010). A holistic approach to wireless sensor network routing in underground tunnel environments. Computer Communications 33(13): 15661573. https://doi.org/10.1016/j.comcom.2010.04.017

[15] Bhattacharjee S, Roy P, Ghosh S, Misra S, Obaidat MS. (2012). Wireless sensor network-based fire detection, alarming, monitoring and prevention system for Bordand-Pillar coal mines. Journal of Systems and Software 85(3):

571-581. https://doi.org/10.1016/j.jss.2011.09.015

[16] Chen W, Jiang X, Li X, Gao J, Xu X, Ding S. (2013). Wireless Sensor Network nodes correlation method in coal mine tunnel based on Bayesian decision. Measurement 46(8): 2335-2340. https://doi.org/10.1016/j.measurement.2013.04.018

[17] Zhou G, Zhu Z, Zhang P, Li W. (2016). Node deployment of band-type wireless sensor network for underground coalmine tunnel. Computer Communications 81: 43-51. https://doi.org/10.1016/j.comcom.2015.10.015 